\title{
Gravitational Waves from The Newtonian plus Hénon-Heiles System*
}

\author{
Fernando Kokubun \\ Departamento de Física, Fundação Universidade do Rio \\ Grande \\ Caixa Postal 474, CEP 96201-900, Rio Grande, RS, Brazil
}

\begin{abstract}
In this work we analyze the emission of gravitational waves from a gravitational system described by a Newtonian term plus a Hénon-Heiles term. The main concern is to analyze how the inclusion of the Newtonian term changes the emission of gravitational waves, considering its emission in the chaotic and regular regime.
\end{abstract}

*Accepted for publication in Phys.Lett.A 
In a previous paper we analyzed the emission of gravitational waves from a HénonHeiles System, showing the qualitative differences between gravitational waves emission from chaotic and regular system [1]. Although interesting, the Hénon-Heiles system is not realistic and it was originally intended to study galactic dynamics, such that the emission of gravitational waves is expected to be negligible. Thus in this work we include a Newtonian term $\propto r^{-1}$ to the Hénon-Heiles potential. This class of potential was analysed by Vieira and Letelier [2], and correspond to a black-hole with external halo. This type of system is more realistic than simple Hénon-Heiles system and its gravitational waves emission may be important due to its stellar system size.

In this work, the dynamics are determined by the gravitational potential $V=$ $-G M m / r+m \omega^{2}\left[\left(x^{2}+y^{2}\right) / 2+\left(x^{2} y-y^{3} / 3\right) / a\right]$. Using the usual approach, we work in a dimensionless system, such that the gravitational potential is reduced to

$$
\Phi=-\frac{\alpha}{\tilde{r}}+\left[\frac{\eta^{2}+\xi^{2}}{2}+\eta^{2} \xi-\frac{\xi^{3}}{3}\right]
$$

with $\Phi \equiv V /\left(m \omega^{2} a^{2}\right), \alpha \equiv G M /\left(\omega^{2} a^{3}\right), \tilde{r} \equiv r / a, \eta \equiv x / a$ and $\xi=y / a$. A main difference between pure Hénon-Heiles [4] and above potential, is that bounded trajectories in the first case are limited to the range $0<E<1 / 6$, whereas in our case we have bounded trajectories even with $E<0$. Other difference is that the upper limit in the energy ( $\left.E_{\max }=1 / 6 \sim 0.167\right)$ may be lower than a pure Hénon-Heiles case, its value depending of the $\alpha$ parameter. For example with $\alpha=0.1$, we have $E_{\max } \sim 0.07$ and with $\alpha=0.01$ we have $E_{\max } \sim 0.156$. With above potential, we calculated the trajectories using numerical simulations, changing energy $E$, the parameter $\alpha$ and initial conditions. For our simulations we used $\alpha=0.01,0.001$ and 0.0001 . The main reason to these choice is to maintain some resemblance with Hénon-Heiles case. (Later in the text, we discuss the physical meaning and limitations of this choice.) Note that with these $\alpha$ we have always $E_{\max } \sim 1 / 6$. We used in our simulations three distinct values for the energies, i.e., $E=0.1,0.01$ and 0.001 . In this range of energy we obtain a good mixture of chaotic and regular motions for our choice of $\alpha$ s, permitting a better comparison between emission of gravitational waves from 
both cases.

The power emitted as a gravitational waves was determined using the standard quadrupole formula

$$
P \equiv \frac{d E}{d t}=\lambda \dddot{q}_{\alpha \beta}^{2}
$$

where

$$
\lambda=\frac{G m}{45 c^{5}} a^{2} \omega^{3}
$$

and quadrupole terms are given by $q_{\eta \eta}=2 \eta^{2}-\xi^{2}, q_{\xi \xi}=2 \xi^{2}-\eta^{2}$ and $q_{\eta \xi}=3 \eta \xi$. (In what follow we use $T$ instead of $\omega$, with $T \equiv 2 \pi / \omega$.)

In the figures 1, 2 and 3 we show some results of our simulations. We plot trajectories in the X-Y plane, Poincaré section and the spectrum of the emitted gravitational waves for $\alpha=0.01$ and three different energies $E=0.10,0.01$ and 0.001 . In each case we plot one chaotic and one regular motion. The maximum power emitted as a gravitational waves in

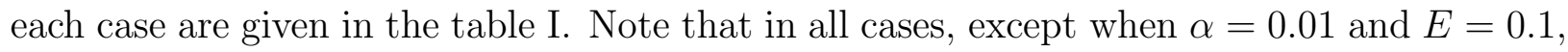
the power emitted in chaotic case is greater than regular case ( This is an exception, as can be seen in the table [V where we show a more detailed statistics of emitted power.).

The irregular characteristic of the gravitational waves spectrum is show in all chaotic case, being more strong with $E=0.1$. In particular note the strong differences of the emitted spectrum of the chaotic and regular case in the figure 1.

In the tables $\llbracket,[\mathrm{II}$ and $\mathrm{IV}$ we show with more details the power emitted as a gravitational waves for several $\alpha$ and $E$. As expected, the emission in the chaotic case is greater than regular case [1]. However, for lower $E$ the differences between regular and chaotic case becomes negligible. This happens because for high $E$, the trajectories reach the boundaries of the Hénon-Heiles term. In this case the trajectories of the particles suffer a strong changes, resulting in a higher emission of gravitational waves. On the other hand for lower $E$, the trajectories are confined far away from Hénon-Heiles boundaries, such that it not suffer strong changes as the previous case. However, in all cases the spectrum of the emitted 
gravitational waves are much more irregular in the chaotic case when compared with the regular case.

As soon the potential which we are using is more realistic than a simple Hénon-Heiles case, it will be interesting to verify in which conditions the emission of gravitational waves will be important. To do it, it is important to fix the values of $\alpha$ and $\lambda$. Thus in order to obtain a better understanding of $\alpha$ and $\lambda$, we assume two typical size for the system: (1) Solar System and (2) binary pulsar (typically PSR $1913+16)$ with parameters $\left(a_{1}, T_{1}\right),\left(a_{2}, T_{2}\right)$ respectively. Then we set $T=T_{o} T_{i}, a=a_{o} a_{i}, i=1,2$ with $a_{1}=1.5 \times 10^{11} \mathrm{~m}, T_{1}=3.1 \times 10^{7} s$, $a_{2}=2.0 \times 10^{9} \mathrm{~m}$ and $T_{2}=2.8 \times 10^{4} \mathrm{~m}$ and $M=\beta_{1} M_{\odot}, m=\beta_{2} M_{\odot}$ with $M_{\odot}=2.0 \times 10^{30} \mathrm{~g}$ (Solar mass). Thus

$$
\begin{gathered}
\text { Solar System size : } \alpha_{1}=0.96 \beta_{1} \frac{T_{o}^{2}}{a_{o}^{3}} ; \lambda_{1}=2.29 \times 10^{-22} \beta_{2} \frac{a_{o}^{2}}{T_{o}^{3}} \\
\text { Binary Sistem size : } \alpha_{2}=0.33 \beta_{1} \frac{T_{o}^{2}}{a_{o}^{3}} ; \lambda_{2}=5.5 \times 10^{-17} \beta_{2} \frac{a_{o}^{2}}{T_{o}^{3}}
\end{gathered}
$$

In the tables $\nabla$ and $\nabla \mathbb{1}$ we show some numerical values for $a_{o}, T_{o}$ and $\lambda$ using $\alpha=$ 0.0001, 0.001 and 0.01 and $\beta_{1}=\beta_{2}=1.4$. Also, we show $\Delta E / E_{i} \sim\left(<P_{\max }>T_{o}\right) / E_{i}$ which is the energy lost as a gravitational waves during a typical crossing time $T_{o}$ and we use two values for $E_{i}$, i.e., $E_{i}=E_{\text {max }}=0.10$ and $E_{i}=E_{\text {min }}=0.001$. This last column show how much the emission are important to the dynamics of the system. Only when $\Delta E / E_{o} \ll 1$ the emission of gravitational waves will be negligible to the dynamics of the system. (Although $\Delta E / E_{o}>1$ is meaningless, we show these values only for the sake of completeness.) From the tables $\mathrm{V}$ and $\mathrm{V1}$ we see that when the system size is of the order of a binary pulsar the emission of gravitational waves will be important. On the other hand when the size is of the order of the Solar System the energy lost as a gravitational waves are negligible only in the cases (Ia) and (Ib). In all other cases the energy lost as a gravitational waves are of the same order of magnitude than its initial energy.

Note that $\Delta E / E$ is lower when $\alpha$ and $E_{o}$ are lower. As said before, in these cases the size of typical trajectory is small compared with the size of external halo. For example when $\alpha=0.0001$ and energies $E_{o}=0.001$ and $E=0.10$ we obtained with numerical simulations 
that $|\xi|_{\max } \sim 0.07$, and $|\xi|_{\max } \sim 0.6$ respectively. These result show that the size of the trajectories with small energy are smaller than trajectories with high energy (as expected). This mean that in the first case (small energy) the trajectories do not reach the boundaries defined by the Hénon-Heiles term, whereas in the high energy case these boundaries can be reached. Thus in the first case (low energy), the external halo is felt only as a small perturbations (note that for small $(\eta, \xi)$ the Hénon-Heiles term is negligible in respect to the Newtonian term).

Therefore a potential like as given by equation 1, is useful only when external halo is felt as a small perturbations (as soon we consider the effect of the gravitational waves to the dynamics of the system).

However, even with inclusion of the Newtonian term, the emission of gravitational waves are greater in the chaotic case than regular case - except for low energy case - , like our previous results [1]. Also the spectrum of the emitted wave is highly irregular, thus we can speculate that with a careful analyze of the gravitational waves spectrum we can detect a gravitational chaotic system. But it is important to note that our results show that if a system is described by a Newtonian potential with a Hénon-Heiles term its typical size need to be greater than a Solar System size. If this is not the case, the emission of gravitational waves will be important and it will collapse quickly. This show that although more realistic, a single inclusion of the Newtonian term is not sufficient as a realistic core-halo system model (see for example [3] for a more realistic approach).

This work was partially supported by Fundação de Amparo a Pesquisa do Rio Grande do Sul (FAPERGS), brazilian financial agency. 


\section{REFERENCES}

[1] F. Kokubun, Phys.Rev.D15,57, 2610-2612, (1998).

[2] W. M. Vieira and P.Letelier, Phys.Rev.Lett.,76 ,1409, (1996).

[3] W. M. Vieira and P.S. Letelier, Phys.Lett A,228,22-24, 1997.

[4] M. Hénon and C.Heiles, Astron.J.,69,73 (1964). 


\section{FIGURES}

FIG. 1. An examples of chaotic and regular motions with $E=10^{-1}$ and $\alpha=10^{-2}$. The left box show the trajectories in the $\mathrm{X}-\mathrm{Y}$ plane, the central box the Poincaré section and the right box the some portion of the gravitaional waves spectrum. The chaotic case is show in the top figures set, and regular case at bottom set.

FIG. 2. Same as previous figure, but now using $E=10^{-2}$.

FIG. 3. Same as previous figure, but now using $E=10^{-3}$. 


\section{TABLES}

\begin{tabular}{llr}
\hline \hline$E$ & $P_{\max } / \lambda$ & \\
\hline 0.100 & $5.21 \times 10^{17}$ & chaotic \\
0.100 & $5.34 \times 10^{17}$ & regular \\
0.010 & $1.49 \times 10^{16}$ & chaotic \\
0.010 & $6.73 \times 10^{15}$ & regular \\
0.001 & $1.04 \times 10^{16}$ & chaotic \\
0.001 & $5.23 \times 10^{15}$ & regular \\
\hline \hline
\end{tabular}

TABLE I. Maximum emiited power as a gravitational waves for the cases shown in the figures 1.,2 and 3

\begin{tabular}{cccc}
\hline \hline$\alpha$ & $\mathrm{E}$ & $P_{\text {max }} / \lambda$ & Note \\
\hline 0.0001 & 0.100 & $(8.15 \pm 3.78) \times 10^{12}$ & chaotic+regular \\
0.0001 & 0.100 & $(5.89 \pm 2.49) \times 10^{12}$ & regular \\
0.0001 & 0.100 & $(1.00 \pm 0.36) \times 10^{13}$ & chaotic \\
0.0001 & 0.010 & $(6.26 \pm 2.74) \times 10^{10}$ & chaotic+regular \\
0.0001 & 0.010 & $(6.43 \pm 2.81) \times 10^{10}$ & regular \\
0.0001 & 0.010 & $(5.65 \pm 2.43) \times 10^{10}$ & chaotic \\
0.0001 & 0.001 & $(2.72 \pm 1.22) \times 10^{9}$ & regular \\
\hline \hline
\end{tabular}

TABLE II. Maximum power emitted as a gravitationala waves with $\alpha=0.0001$ for three energies $E=0.1,0.01$ and 0.001 


\begin{tabular}{cccc}
\hline \hline$\alpha$ & $\mathrm{E}$ & $P_{\max } / \lambda$ & Note \\
\hline 0.0010 & 0.100 & $(1.53 \pm 0.73) \times 10^{17}$ & chaotic+regular \\
0.0010 & 0.100 & $(9.61 \pm 5.44) \times 10^{16}$ & regular \\
0.0010 & 0.100 & $(1.78 \pm 0.66) \times 10^{17}$ & chaotic \\
0.0010 & 0.010 & $(2.09 \pm 0.96) \times 10^{15}$ & chaotic+regular \\
0.0010 & 0.010 & $(1.25 \pm 0.45) \times 10^{15}$ & regular \\
0.0010 & 0.010 & $(2.31 \pm 0.94) \times 10^{15}$ & chaotic \\
0.0010 & 0.001 & $(4.97 \pm 2.41) \times 10^{14}$ & chaotic+regular \\
0.0010 & 0.001 & $(5.00 \pm 2.60) \times 10^{14}$ & regular \\
0.0010 & 0.001 & $(4.87 \pm 1.70) \times 10^{14}$ & chaotic \\
\hline \hline
\end{tabular}

TABLE III. Maximum power emitted as a gravitationala waves with $\alpha=0.001$ for three energies $E=0.1,0.01$ and 0.001

\begin{tabular}{cccc}
\hline \hline$\alpha$ & $\mathrm{E}$ & $P_{\max } / \lambda$ & Note \\
\hline 0.0100 & 0.100 & $(2.55 \pm 0.79) \times 10^{17}$ & chaotic+regular \\
0.0100 & 0.100 & $(1.41 \pm 0.59) \times 10^{17}$ & regular \\
0.0100 & 0.100 & $(2.60 \pm 0.76) \times 10^{17}$ & chaotic \\
0.0100 & 0.010 & $(1.76 \pm 0.82) \times 10^{16}$ & chaotic+regular \\
0.0100 & 0.010 & $(1.38 \pm 0.62) \times 10^{16}$ & regular \\
0.0100 & 0.010 & $(1.83 \pm 0.84) \times 10^{16}$ & chaotic \\
0.0100 & 0.001 & $(1.07 \pm 0.57) \times 10^{16}$ & chaotic+regular \\
0.0100 & 0.001 & $(8.69 \pm 3.80) \times 10^{15}$ & regular \\
0.0100 & 0.001 & $(1.56 \pm 0.65) \times 10^{16}$ & chaotic \\
\hline \hline
\end{tabular}

TABLE IV. Maximum power emitted as a gravitationala waves with $\alpha=0.01$ for three energies $E=0.1,0.01$ and 0.001 . 


\begin{tabular}{|c|c|c|c|c|c|c|}
\hline & $\alpha$ & $a_{o}$ & $T_{o}$ & $\lambda$ & $\Delta E / E_{\max }$ & $\Delta E / E_{\min }$ \\
\hline (Ia) & 0.0001 & 1 & $8.63 \times 10^{-3}$ & $4.99 \times 10^{-16}$ & $4.32 \times 10^{-4}$ & $1.21 \times 10^{-5}$ \\
\hline (Ib) & 0.0001 & 23.7 & 1 & $1.30 \times 10^{-19}$ & $5.0 \times 10^{-5}$ & $3.5 \times 10^{-7}$ \\
\hline (Ic) & 0.0010 & 1 & $3.23 \times 10^{-2}$ & $1.21 \times 10^{-17}$ & $6.94 \times 10^{-1}$ & $1.87 \times 10^{-1}$ \\
\hline (Id) & 0.0010 & 9.80 & 1 & $2.80 \times 10^{-20}$ & $4.98 \times 10^{-2}$ & $1.34 \times 10^{-2}$ \\
\hline (Ie) & 0.0100 & 1 & $8.63 \times 10^{-2}$ & $4.99 \times 10^{-19}$ & $1.12 \times 10^{-1}$ & $4.31 \times 10^{-1}$ \\
\hline (If) & 0.0100 & 5.12 & 1 & $8.39 \times 10^{-21}$ & $2.18 \times 10^{-2}$ & $8.39 \times 10^{-2}$ \\
\hline
\end{tabular}

TABLE V. Some set of values for $a_{o}, T_{o}$ and $\lambda$ when typical size correspond to our Solar System with $a_{1}=1.5 \times 10^{11} \mathrm{~m}$ and $T_{2}=3.1 \times 10^{7} \mathrm{~s}$ (see text). In the last two columns, we uses $E_{\max }=0.10$ and $E_{\text {min }}=0.001$

\begin{tabular}{lcccccc}
\hline \hline & $\alpha$ & $a_{o}$ & $T_{o}$ & $\lambda$ & $\Delta E / E_{\max }$ & $\Delta E / E_{\min }$ \\
\hline (IIa) & 0.0001 & 1 & $1.47 \times 10^{-2}$ & $2.42 \times 10^{-11}$ & $3.56 \times 10^{1}$ & $9.67 \times 10^{-1}$ \\
$(\mathrm{IIb})$ & 0.0001 & 16.7 & 1 & $2.12 \times 10^{-14}$ & 2.12 & $5.77 \times 10^{-2}$ \\
$(\mathrm{IIc})$ & 0.0010 & 1 & $5.51 \times 10^{-2}$ & $3.31 \times 10^{-13}$ & $3.25 \times 10^{3}$ & $8.76 \times 10^{3}$ \\
$(\mathrm{IId})$ & 0.0010 & 6.91 & 1 & $2.63 \times 10^{-15}$ & $4.68 \times 10^{3}$ & $1.26 \times 10^{3}$ \\
$(\mathrm{IIe})$ & 0.0100 & 1 & 0.14 & $2.45 \times 10^{-14}$ & $4.75 \times 10^{5}$ & $1.7 \times 10^{6}$ \\
(IIf) & 0.0100 & 3.59 & 1 & $9.92 \times 10^{-16}$ & $2.6 \times 10^{3}$ & $1.0 \times 10^{4}$ \\
\hline \hline
\end{tabular}

TABLE VI. Some set of values for $a_{o}, T_{o}$ and $\lambda$ when typical size correspond to a bynary pulsar with $a_{2}=2.0 \times 10^{9} \mathrm{~m}$ and $T_{2}=2.8 \times 10^{4} \mathrm{~s}$ (see text). In the last two columns, we uses $E_{\max }=0.10$ and $E_{\text {min }}=0.001$ 
$\alpha=10^{-2} \quad E=10^{-1}$

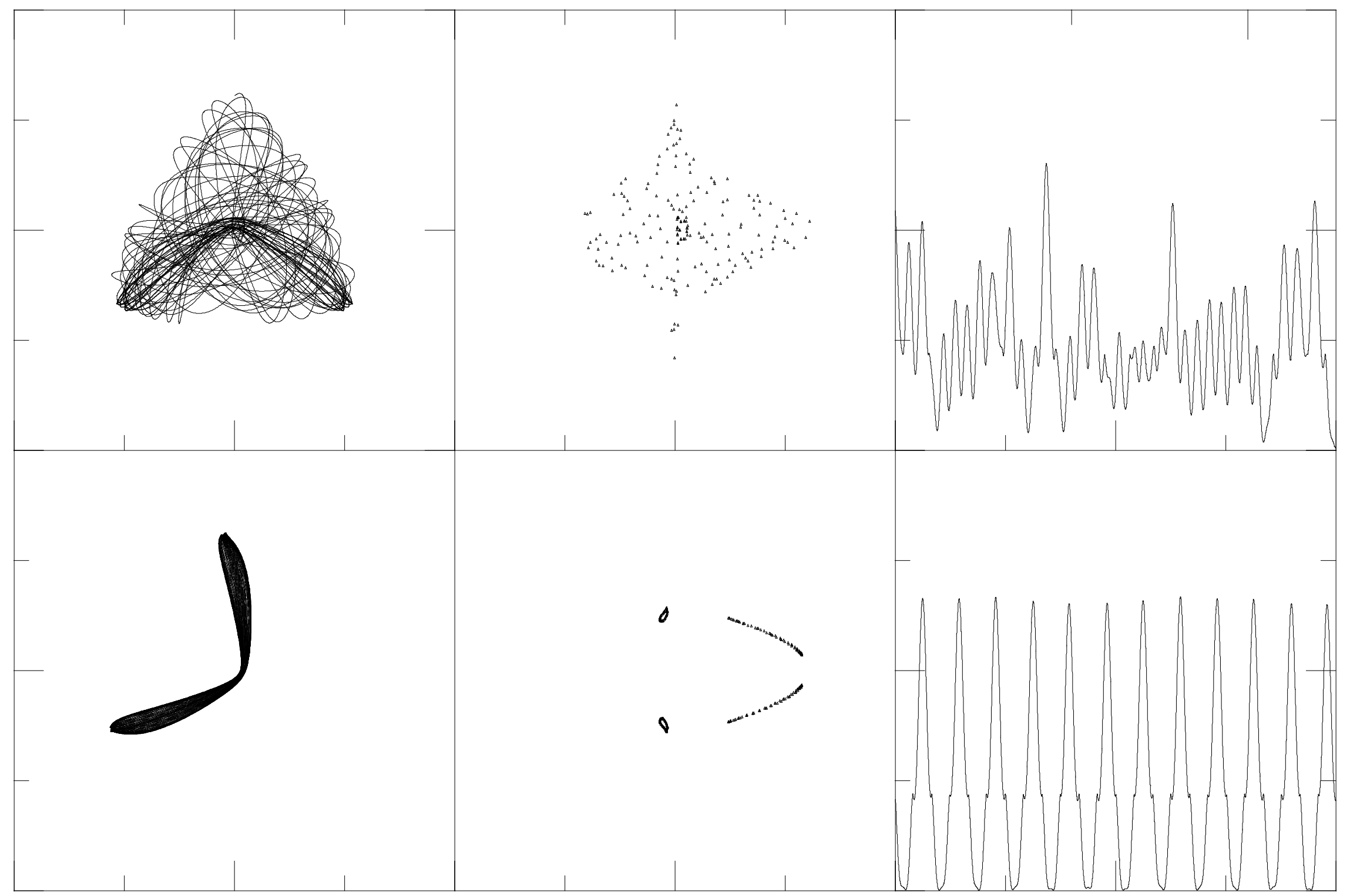

Figure 1

"Gravitational Waves from The Newtonian plus Henon-Heiles System" by Fernando Kokubun 
$\alpha=10^{-2} \quad E=10^{-2}$

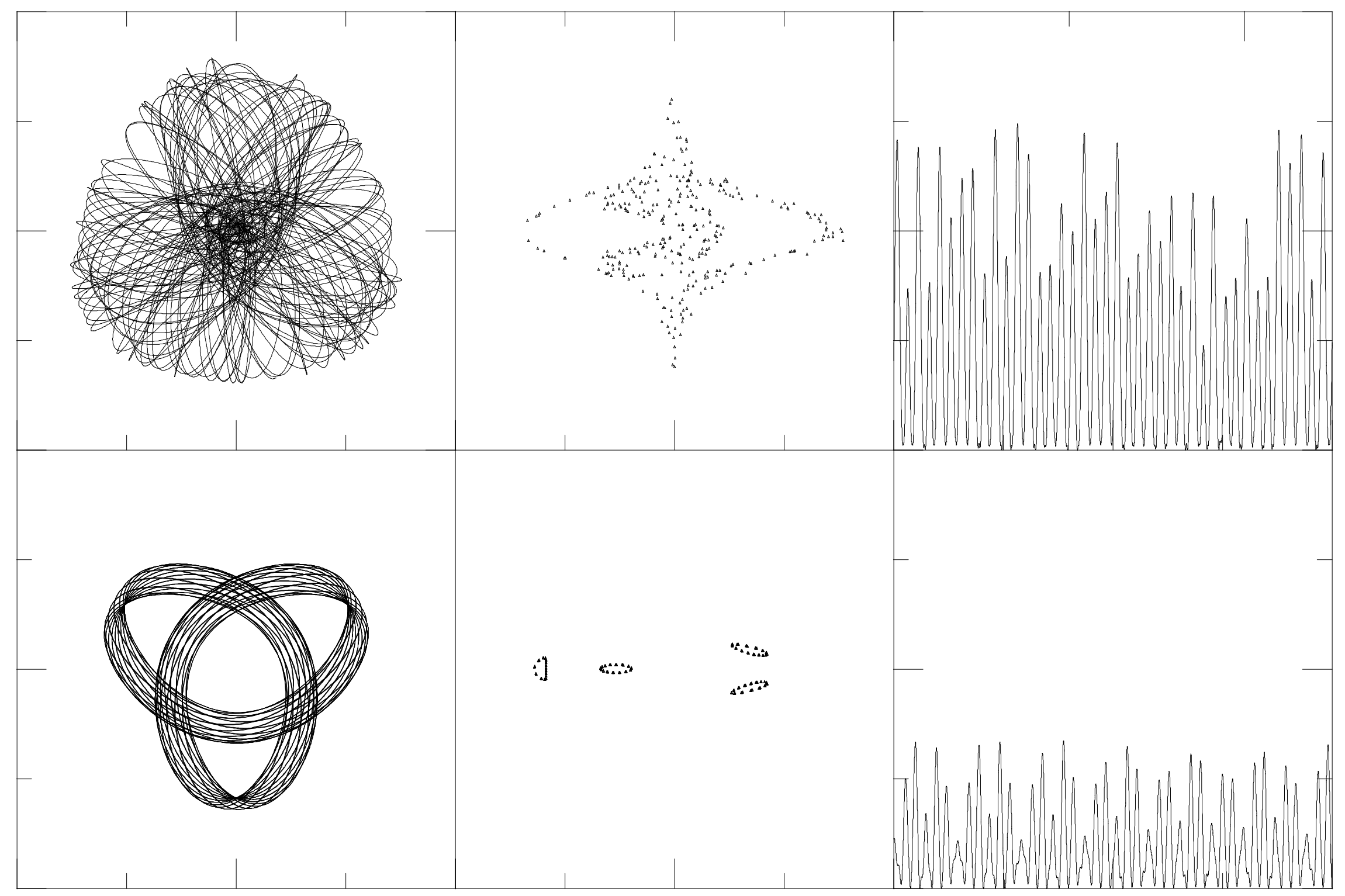

Figure 2

"Gravitational Waves from The Newtonian plus Henon-Heyles System" by Fernando Kokubun 
$\alpha=10^{-2} \quad E=10^{-3}$

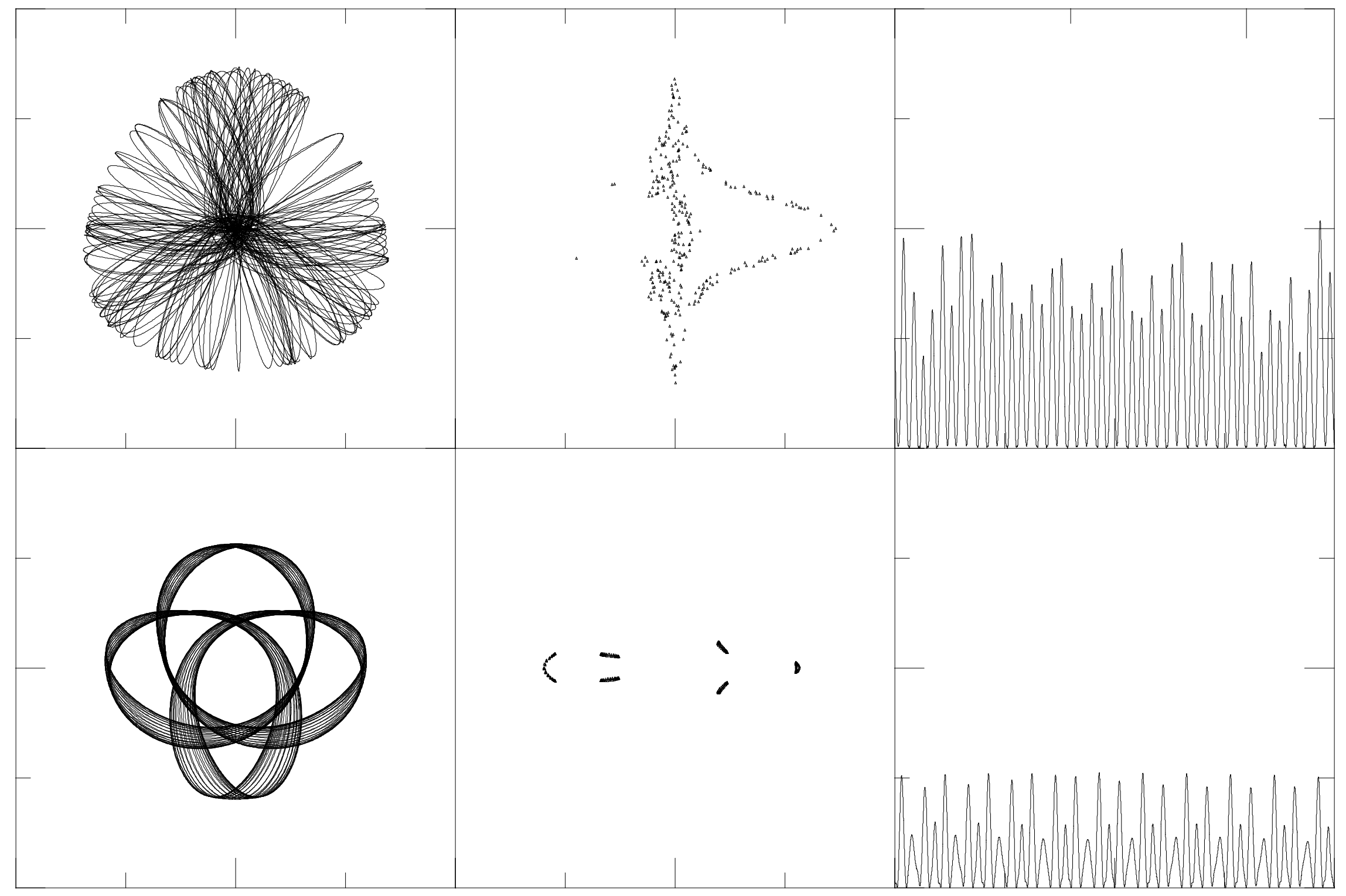

Figure 3

"Gravitational Waves from The Newtonian plus Henon-Heiles System"

by Fernando Kokubun 